\title{
Immune-Inflammatory Cell Profile and Receptor Activator of Nuclear Factor Kappa B Ligand/Osteoprotegerin Expression in Persistent Apical Periodontitis after Root Canal Retreatment Failure
}

\author{
Carlos Estrela, DDS, MSc, PhD, ${ }^{*}$ Daniel de Almeida Decurcio, DDS, MSc, PhD, * \\ Júlio Almeida Silva, DDS, MSc, PbD, * Aline Carvalbo Batista, DDS, MSc, PbD, * \\ Nathália Caroline de Souza Lima, DDS, MSc, ${ }^{\dagger}$ Brunno Santos de Freitas Silva, DDS, MSc, PhD, * \\ João Antonio Chaves de Souza, DDS, MSc, PhD, ${ }^{\neq}$and Carlos Alberto Souza Costa, DDS, MSc, PbD
}

\section{Ahstract}

Introduction: This study assessed the immuneinflammatory profile and the expression of bone resorption activators receptor activator of nuclear factor kappa $B$ ligand (RANKL) and inhibitor osteoprotegerin (OPG) in apical periodontitis $(n=20)$ that persisted after root canal retreatment. Methods: Immunohistochemistry was used to characterize lymphocyte populations $\left(\mathrm{CD}^{+}{ }^{+}\right.$, $\mathrm{CD}^{2} 5 \mathrm{RO}^{+}, \mathrm{CD}^{+}$, and FoxP3 ${ }^{+}$cells), macrophages $\left(\mathrm{CD}^{+} 8^{+}\right), \mathrm{RANKL}^{+}$and $\mathrm{OPG}^{+}$cells in persistent apical periodontitis (PAP) and primary periapical lesions (PPLs). By using quantitative real-time polymerase chain reaction, the mRNA expression of RANKL and OPG in PAP and periodontal ligament from healthy teeth was comparatively analyzed. The data were analyzed by Mann-Whitney, Pearson $\chi^{2}$, and Wilcoxon tests $(5 \%$ level). Results: PAP showed an elevated number of FoxP3 ${ }^{+}$cells compared with PPL $(P<.001)$. The number of $\mathrm{CD}^{+} 8^{+}$cells was reduced in the PAP samples compared with the PPLs $(P<.001)$. Similar number of other lymphocyte populations was observed in PAP and PPLs ( $P>.05$ for all comparisons). No differences in the RANKL, OPG, and immune-inflammatory cells were demonstrated when comparing PAP microscopically classified as cyst with those classified as granulomas ( $P>.05$ for all comparisons). The assessment of $m$ RNA expression revealed higher levels of RANKL and OPG in PAP compared with the periodontal ligament from healthy teeth (control) samples $(P<.001)$. Also, a greater expression of RANKL in comparison with OPG was observed in PAP $(P<.001)$. Conclusions: These findings indicate that PAP consists of biologically active lesions that demonstrate potential of bone resorption (higher expression of RANKL) and is characterized by an immune-inflammatory cell profile that suggests a suppressive and regulatory environment (higher number of FoxP3 ${ }^{+}$cells and lower number of macrophages) favorable to more chronic clinical behavior. (J Endod 2016;42:439-446)

\section{Key Words}

Apical periodontitis, bone resorption, immunological cells, osteoprotegerin, RANK ligand

$\Lambda$ pical periodontitis (AP) from endodontic origin is an inflammatory response to Abacteria in the root canal system. Primary periapical lesions (PPLs) appear as an initial response of the organism to the destruction of dental pulp and root canal infection, whereas secondary lesions occur after root canal treatment fails (1). Periapical lesions (PLs) after root canal retreatment are classified as persistent in the presence of intraradicular infection or extraradicular factors that may affect healing.

Inflammatory responses are nonspecific mechanisms that consist of immediate vascular exudative and proliferative phenomena as well as proliferation and infiltration of inflammatory cells and that destroy, dilute, or circumscribe pathogenic agents in tissue defense (2). Immune responses are complex and involve the processing, presentation, and recognition of antigens that begin in the dental pulp $(2,3)$. Thus, PLs consist of an initial inflammatory reaction, followed by the recruitment of polymorphonuclear neutrophils, macrophages, and dendritic cells (4). In the chronic stage of the disease, various T-lymphocyte subsets are found (5-7).

Periapical inflammation usually stimulates the formation of granulomas or cysts infiltrated by nonspecific and specific cells involved in local immune reactions. Most cells in these lesions are T and B lymphocytes and macrophages (8). T cells, which are essential mediators of leukocyte recruitment, are implicated in responses to PL pathogens. T lymphocytes are part of the PL inflammatory infiltrate; $\mathrm{CD}^{+} \mathrm{T}$ lymphocytes, $\mathrm{CD} 45 \mathrm{RO}^{+}$memory T lymphocytes, $\mathrm{CD}^{+}$cytotoxic $\mathrm{T}$ lymphocytes, and $\mathrm{FoxP} 3^{+}$:regulatory $\mathrm{T}$ lymphocytes are sometimes found in periapical cysts and granulomas, which suggests the existence of a specific immune response in those lesions (9-11).

Granulomas and periapical cysts promote the resorption of bone tissue adjacent to the root apex. The receptor activator of nuclear factor kappa B ligand (RANKL) and

From the *Department of Stomatologic Sciences, Federal University of Goiás, Goiânia, Goiás, Brazil; ${ }^{\dagger}$ University of Campinas, Piracicaba, São Paulo, Brazil; and ${ }^{\ddagger}$ Department of Physiology and Pathology, São Paulo State University, Araraquara School of Dentistry, Araraquara, São Paulo, Brazil.

Address requests for reprints to Prof Dr Carlos Estrela, Department of Stomatologic Sciences, Federal University of Goiás, Praça Universitária s/n, Setor Universitário CEP 74605-220, Goiânia, GO, Brazil. E-mail address: estrela3@terra.com.br

0099-2399/\$ - see front matter

Copyright (C) 2016 American Association of Endodontists.

http://dx.doi.org/10.1016/j.joen.2015.11.012 
osteoprotegerin (OPG) expressed in AP play an important role in the development of these lesions (12). The interaction of RANK and RANKL is necessary for the activation and differentiation of osteoclasts, an event regulated by OPG that, in turn, inhibits osteoclast differentiation by preventing the interaction of RANK and RANKL (13). Changes in the balance of these mediators define the severity and progression of some bone diseases such as those found in periodontal and periapical lesions (14).

In addition to its role in the differentiation and activity of osteoclasts, the RANK-RANKL system may also affect immune-inflammatory responses. RANKL expression is induced by activated $\mathrm{T}$ cells, which downregulate the production of proinflammatory cytokines both in vivo and in vitro in response to stimulation by bacteria and their products (15). Higher levels of FoxP3 (Treg cells) and RANKL are found in granulomas (16). Th17, a T-cell subtype that regulates osteoclastogenesis and bone resorption, has been implicated in PL formation and in RANKL induction and consequent OPG decoy $(17,18)$. Th17 and regulatory T cells (Treg cells) participate in immune cell homeostasis and play an important role in the induction and maintenance of immune tolerance (19). The forkhead box P3 (FoxP3) transcription factor is a crucial controller of Treg cells with some evidence of activity on osteoclasts in periapical cysts and granulomas (17). In a recent study (20), differential methylation patterns of FoxP3 in PLs seemed to be crucial to determine their suppressive activity, which might be involved in PL development.

Endodontists should be aware of disease onset, response, and healing in persistent apical periodontitis (PAP) associated with the failure of root canal retreatment. Knowledge of the complex response in PAP leads to better understanding of diagnosis, prognosis, and treatment planning. Therefore, immune-inflammatory responses of granulomas, periapical cysts, and primary and secondary apical periodontitis should be carefully investigated. However, only a few clinical studies have described the immunologic factors involved in PAP. Our study analyzed the immune-inflammatory cells profile and the expression of the bone resorption modulators (RANKL and OPG) in AP that persisted after root canal retreatment.

\section{Sample Selection}

\section{Materials and Methods}

Twenty PL samples from patients referred to the Endodontic Clinic of the School of Dentistry, Federal University of Goiás (Goiânia, Brazil) for periapical surgery were selected from 2009 to 2011. Patient mean age was 32 years, and 12 were men. All patients underwent clinical and imaging examinations, and their clinical histories were reviewed. The experimental group included clinically healthy patients with a diagnosis of PAP characterized by asymptomatic PLs that persisted for more than 6 months after root canal retreatment without sinus tract involvement or radiographic signs of remission. The clinical selected cases were retreated by the same specialist in endodontics (with more than 10 years of experience) and properly restored. Cases with accidents or complications were not included in the present sample. All patients included in this study were considered clinically healthy with no history of systemic diseases.

The distribution of samples for the immunohistochemistry and quantitative reverse transcription real-time polymerase chain reaction (qRT-PCR) analyses is shown in Figure 1. PLs related with endodontically untreated teeth (PPLs, $n=10$ ) were also included in this study as a comparative group. The PPL samples were obtained from the Oral Pathology Laboratory at the Federal University of Goiás. Periodontal ligaments (qRT-PCR control, $n=10$ ) were obtained from extracted erupted healthy teeth (without caries or periodontal disease) for orthodontic reasons. This study was approved by the Research Ethics Committee of the institution where it was conducted, and all participants signed an informed consent form.

\section{Study Design}

This experimental study conducted immunohistochemical analyses of CD3, CD8, CD45R0, FoxP3, CD68, RANKL, and OPG and the quantitative expression of RANKL and OPG by qRT-PCR by using PAP specimens.

The samples were obtained at the time of periapical surgery (experimental group) or tooth extraction (control group). The lesions were removed only after surgery was indicated because of clinical or radiographic findings. Samples were then divided into 2 parts; part of each specimen was immersed in Trizol reagent (Life Technologies, Grand Island, NY) and stored at $-80^{\circ} \mathrm{C}$ (MDF-C8V; Sanyo Scientific, Bensenville, IL), and the other part was fixed in neutral buffered formalin, embedded by routine technique in paraffin wax, and cut into 5- $\mu \mathrm{m}$ sections for hematoxylin-eosin staining to confirm the diagnosis of PLs.

\section{Immunohistochemistry}

Paraffin-embedded tissues were sectioned $(3 \mu \mathrm{m})$ and collected in series on glass slides coated with $2 \% 3$-aminopropyltriethoxysilane (Sigma-Aldrich, St Louis, M0). The sections were deparaffinized in xylene and rehydrated in decreasing concentrations of alcohol. For antigen retrieval, sections that received the antibodies anti-CD3, anti-CD8, anti-CD45R0, and anti-CD68 were immersed in a solution of $10 \mathrm{mmol} / \mathrm{L}$ monohydrated citrate buffer solution (pH 6.0) and heated in a water bath at $95^{\circ} \mathrm{C}$ for 30 minutes. Slides that received the antibody antiFoxP3, anti-RANKL, and anti-OPG were immersed in a solution containing $1.21 \mathrm{~g}$ TRIS, $0.3722 \mathrm{~g}$ EDTA, and $500 \mu \mathrm{L}$ Tween (pH 9.0) and kept in a water bath at $95^{\circ} \mathrm{C}$ for 30 minutes. Endogenous peroxidase

Persistent Apical Periodontitis

$(N=20)$

\begin{tabular}{|c|c|c|c|c|}
\hline $\begin{array}{c}\text { Control } \\
\text { (Primary Periapica } \\
\text { Lesions; } \mathrm{N}=10 \text { ) }\end{array}$ & \multicolumn{2}{|c|}{$\begin{array}{l}\text { Immunohistochemistry } \\
\qquad(\mathrm{N}=20)\end{array}$} & $\begin{array}{c}\text { PCR } \\
(N=20)\end{array}$ & $\begin{array}{c}\text { Control } \\
\text { (Periodontal } \\
\text { ligament; } \mathrm{N}=10 \text { ) }\end{array}$ \\
\hline $\begin{array}{l}\mathrm{CD}^{+} \\
\mathrm{CD}^{+} 5 \mathrm{RO}^{+} \\
\mathrm{CD}^{+}\end{array}$ & $\begin{array}{l}\text { FoxP3 }^{+} \\
\text {CD68 }^{+}\end{array}$ & $\begin{array}{l}\text { RANKL } \\
\text { OPG }\end{array}$ & \multicolumn{2}{|c|}{$\begin{array}{c}\text { RANKL } \\
\text { OPG }\end{array}$} \\
\hline
\end{tabular}

Figure 1. Distribution of samples for immunohistochemistry and qRT-PCR analyses. 
quenching was blocked with $6 \%$ hydrogen peroxide and a methanol solution in 2 baths of 15 minutes each at room temperature.

After washing with Tris buffer ( $\mathrm{pH} 7.4$ ), the slides were incubated with the following primary antibodies diluted in $1 \%$ phosphate-buffered saline-bovine serum albumin: anti-CD3 (F7.2.38; Dako Co, Glostrup, Denmark; dilution 1:200), anti-CD8 (M7103; Dako Co; dilution 1:200), anti-CD45R0 (UCHL1; Dako Co; dilution 1:200), anti-CD68 (M0876; Dako Co; dilution 1:500), anti-FoxP3 (Biolegend, San Diego, CA; dilution 1:100), anti-RANKL (N-19; Santa Cruz Biotechnology, Santa Cruz, CA; dilution 1:200), and anti-OPG (N20; Santa Cruz Biotechnology; dilution 1:200). The negative control was obtained by omitting the specific primary antibody during the reaction; the positive control for RANKL and OPG was a central giant-cell granuloma. All slides were exposed to an avidin-biotin complex (LSAB-Kit + HRP; Dako Cytomation, Carpinteria, CA) and to 3,3'-diaminobenzidine chromogen (DAB+; Dako Cytomation) and were counterstained with Meyer hematoxylin, dehydrated in ethanol, cleared in xylene, and mounted.

The quantitative analysis was based on the number of positive cells for CD3, CD8, CD45R0, FoxP3, and CD68. Cells were counted (cells/ $\mathrm{mm}^{2}$ ) under a light microscope by using an integration graticule (Netzmikrometer $\times 12.5$; Carl Zeiss, Göttingen, Germany) in 10 alternate high-power fields under $\times 400$ magnification. A total area of $0.3125 \mathrm{~mm}^{2}$ was evaluated for each sample. Only microscopic fields with strong staining were selected for analysis.

RANKL and OPG immunoexpression was evaluated according to the percentage of positive staining cells in relation to the whole area under examination. For RANKL and OPG analysis, staining was scored partially on the basis of the methods described by Gori et al (21) and Hiraishi et al (22), who classified the percentage of positive cells into 5 categories: no staining $=0,1 \%-24 \%=1,25 \%-49 \%=2,50 \%-$ $74 \%=3$, and $>75 \%=4$. The immunohistochemical sections were analyzed by 2 independent, blinded, and calibrated observers under light microscopy at 3 different times.

\section{QRT-PQR}

Total RNA was extracted from 20 PLs by using a Trizol reagent according to the manufacturer's protocol (Invitrogen Corp, Carlsbad, CA). In 8 samples it was not possible to obtain cDNA with reliable quality to perform qRT-PCR. Complementary DNA was synthesized by reverse transcription of $1 \mu \mathrm{g}$ total RNA from tissue samples following manufacturer's recommendations (TaqMan Reverse Transcription Reagents Kit; Applied Biosystems, Foster City, CA). The qPCR reactions were performed in $20 \mu \mathrm{L}$ TaqMan qPCR master mix (Applied Biosystems), diluted cDNA, deionized water, and human-specific pre-designed and optimized primers and probe (TaqMan gene expression assays) (Table 1). The cycling conditions used for all primers were preoptimized: $50^{\circ} \mathrm{C}$ for 2 minutes and 40 cycles at $95^{\circ} \mathrm{C}$ for 15 seconds and $60^{\circ} \mathrm{C}$ for 1 minute. The relative levels of gene expression were determined by using the cycle threshold method and were normalized to the housekeeping gene $\beta$-actin. Results were described as mean mRNA expression from duplicate measurements normalized by internal control ( $\beta$-actin) and as fold change over the levels of expression of the normalized target gene determined in cDNA samples prepared from periodontal ligaments of teeth extracted for orthodontic purposes from 10 patients with good oral health.

\section{Statistical Analysis}

The statistical analysis was performed with software SSPS 17.0 for Windows (Chicago, IL) and GraphPad Prism 6 (GraphPad Inc, San Diego, CA). Because the Shapiro-Wilk test presented a non-normal numeric data distribution, the Mann-Whitney test was used to make a
TABLE 1. Primers Collections and Pre-optimized TaqMan Probes for RT-PCR (TaqMan gene expression assays, Applied Biosystems)

\begin{tabular}{lccc}
\hline $\begin{array}{l}\text { Target } \\
\text { genes }\end{array}$ & Assay ID & Accession no. & $\begin{array}{c}\text { Amplicon } \\
\text { (base pairs) }\end{array}$ \\
\hline RANKL & Hs00243522_m1 & NM_003701.3 & 67 \\
OPG & Hs00900358_m1 & NM_002546.3 & 74 \\
\hline
\end{tabular}

comparative analysis in the independent groups. The unpaired Student $t$ test was used to check differences in immunohistochemical expression of CD3, CD8, CD45R0, FoxP3, and CD68 between PAP and PPL. The same statistical analysis evaluated differences in qRT-PCR expression of RANKL and OPG between PAP and PPL. The difference in RANKL and OPG levels in the same group was assessed by Wilcoxon test.

The Pearson $\chi^{2}$ test was used for the statistical analysis of immunohistochemical expression of RANKL and OPG (score). Results were described as means \pm standard error of means. The level of significance was set at $P<.05$.

\section{Results}

Inflammatory cells and osteoclastogenesis mediators were compared between PAP and PPL. Of the PAP specimens selected, 10 were microscopically diagnosed as periapical cysts and 10 as periapical granulomas. In the control group (PPL), 4 lesions were diagnosed as granulomas and 6 as periapical cysts.

\section{Analysis of Inflammation Mediators}

Immunohistochemical reactions for the inflammatory cell markers revealed a higher expression of $\mathrm{FoxP}^{+}$cells in PAP than in PPL samples $(P<.001)$ (Fig. 2$)$. On the other hand, the number of $\mathrm{CD} 8^{+}$cells was reduced in the PAP samples compared with the PPL $(P<.001)$. Similar number of other lymphocyte populations $\left(\mathrm{CD}^{+}\right.$, CD45RO ${ }^{+}$, and $\mathrm{CD}^{+}$cells $)$was observed in PAP and PPL $(P>.05$ for all comparisons), although the data show a slight downward trend in the number of these T lymphocytes in persistent lesions (Fig. 3). No differences in the immune-inflammatory cells were shown when comparing PAP microscopically classified as cyst with those classified as granulomas ( $P>.05$ for all comparisons) (Fig. 3).

\section{Analysis of Osteoclastogenesis Mediators}

Our results showed that RANKL and OPG mRNA expression and RANKL/OPG ratio were significantly higher in PLs than in healthy periapical tissues $(P<.001)$. However, no differences between cysts and granulomas were found (Fig. 4).

In agreement with mRNA data, immunohistochemical analysis of bone remodeling regulatory molecules revealed positive immunoreactivity to RANKL and OPG (Fig. 5).

RANKL and OPG immunohistochemical scores are shown in Figure 6. The assessment of immunohistochemical expression of RANKL and OPG revealed no statistically significant differences between PAP and PPL or between cysts and granulomas. However, mRNA levels of RANKL and OPG genes were higher in PAP specimens $(P<.001)$, with a greater expression of RANKL than of OPG (Fig. 5).

\section{Discussion}

This study was planned to assess immune-inflammatory cell profile and bone resorption modulators in PAP after the failure of root canal retreatment. Our purpose was to study the association between persistent infection with immune cell response and osteoclastogenesis. 

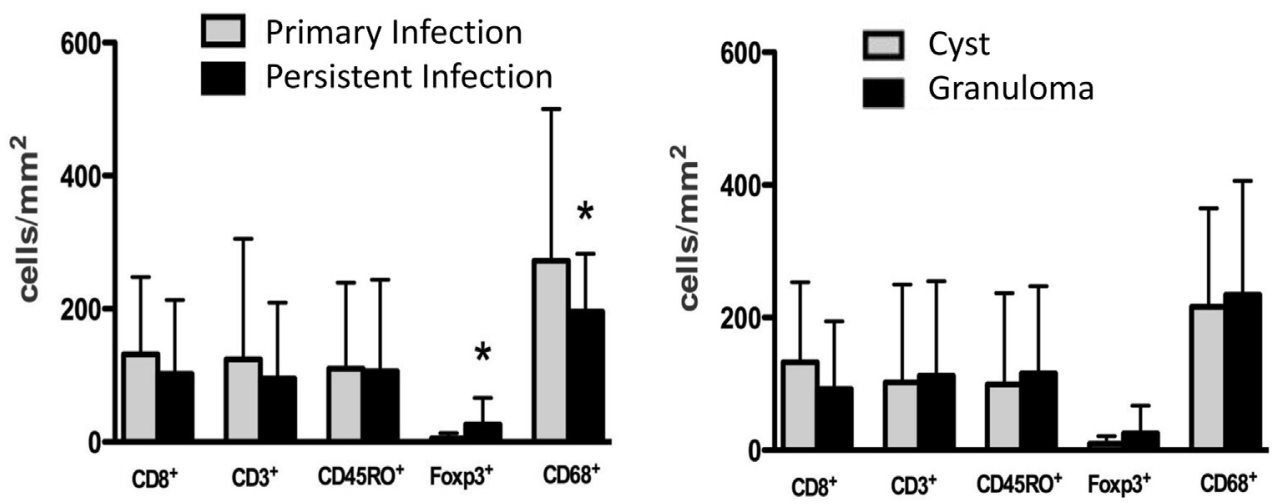

Figure 2. Density of inflammatory cells. Immunohistochemical analysis of $\mathrm{CD}^{+}:$cytotoxic T lymphocytes; $\mathrm{CD}^{+}: \mathrm{T}$ lymphocytes; $\mathrm{CD}^{4} 5 \mathrm{RO}^{+}:$memory $\mathrm{T}$ lymphocytes; $\mathrm{FoxP}^{+}$: regulatory T lymphocytes; $\mathrm{CD}^{+} 8^{+}$: macrophages. *Statistically significant difference $(P<.001)$.

Microbial etiologic factors have been described as responsible for diseases after root canal treatment. According to Nair (1), extraradicular infections are caused by exacerbating AP lesions, periapical actinomycosis, and pieces of infected root dentin displaced into the periapex during root canal instrumentation or cut off from the rest of the root by massive apical resorption and infected periapical cysts, particularly in periapical pocket cysts with cavities open to the root canal.

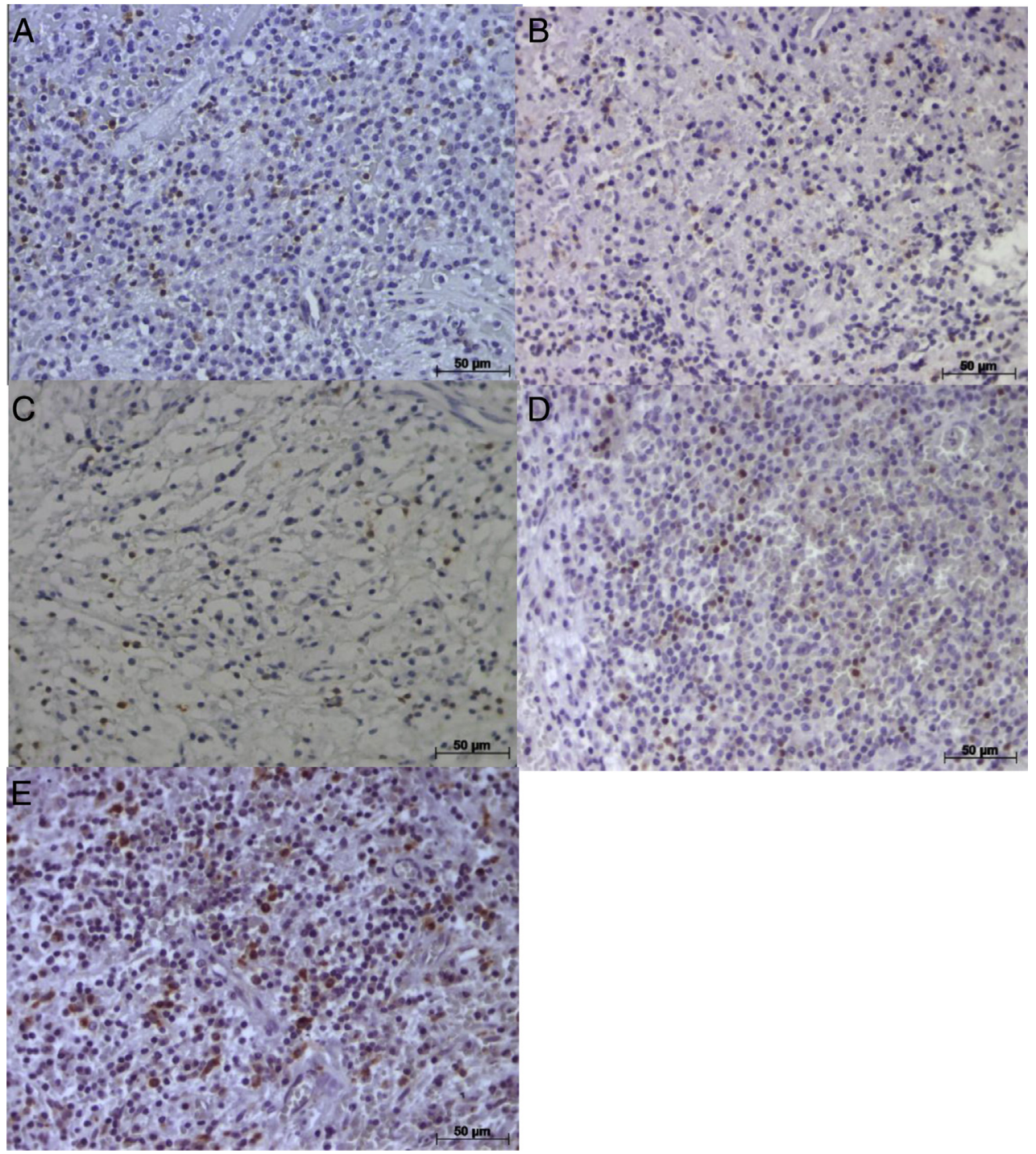

Figure 3. $(A-E)$ Representative photomicrographs of immunohistochemical staining (dark brown) of $\mathrm{CD}^{+}(A), \mathrm{CD}^{+}(B), \mathrm{CD}^{4} 5 \mathrm{RO}^{+}(C), \mathrm{FoxP}^{+}(D)$, and $\mathrm{CD}^{+} 8^{+}(E)$ cells in PAP (original magnification, $\left.\times 400\right)$. 
RANKL mRNA expression

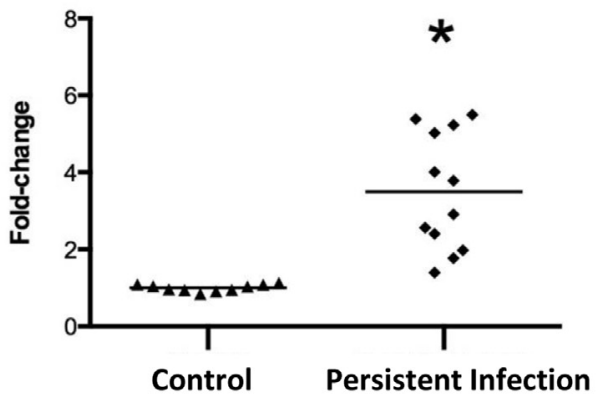

RANKL/OPG

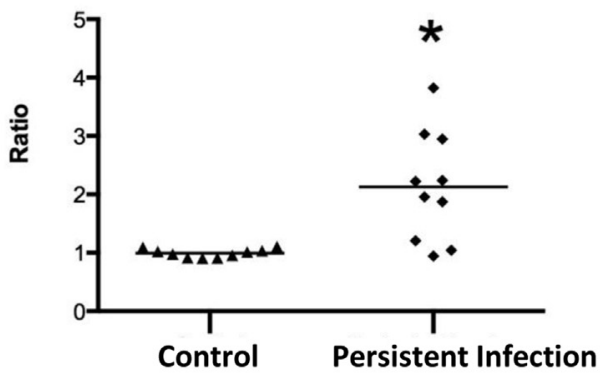

OPG mRNA expression

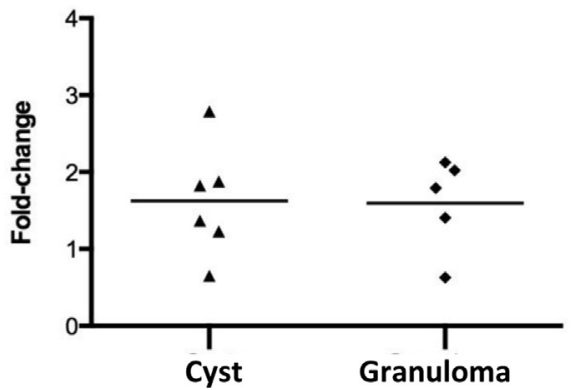

OPG mRNA expression

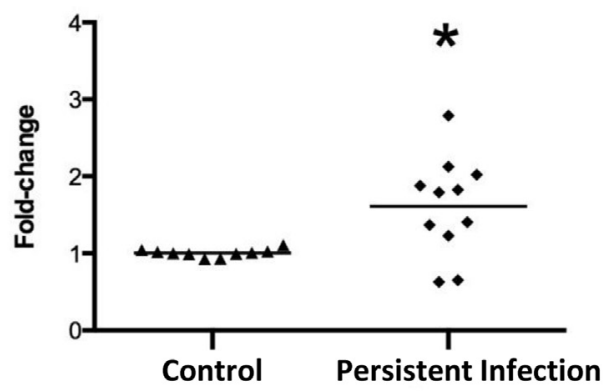

RANKL mRNA expression

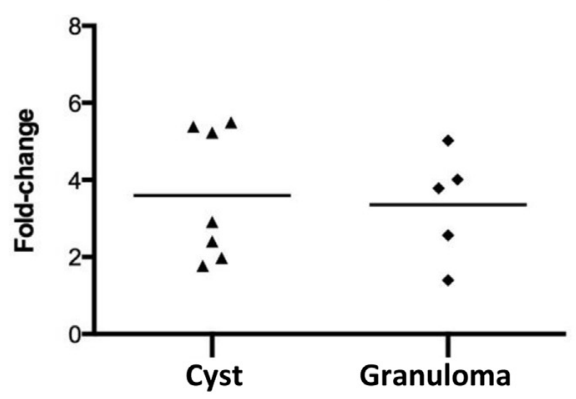

RANKL/OPG

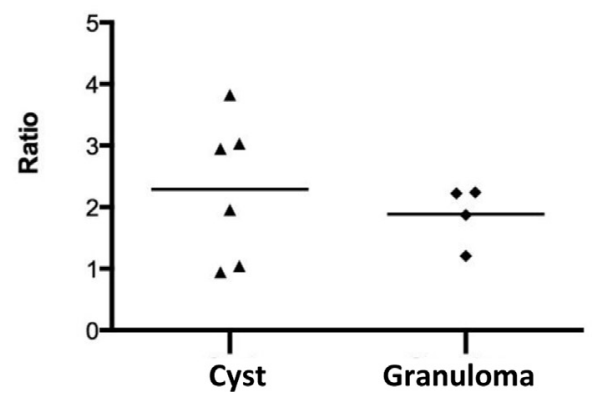

Figure 4. Expression of RANKL and OPG in PAP. RT-PCR showed significant difference in gene expression among control and PAP groups. No difference between cyst and granuloma was found. The results are presented as an expression of the individual mRNAs, with normalization to the housekeeping gene by using the cycle threshold method. *Statistically significant difference between groups.

PAP characterizes a clinical condition of intraradicular microbial resistance to root canal retreatment or extraradicular infection. All lesions analyzed in this study were obtained from selected clinically healthy patients with asymptomatic lesions that persisted for more than 6 months after root canal retreatment without sinus tract involvement, or that persisted for more than 6 months after root canal retreatment and had no radiographic signs of remission.

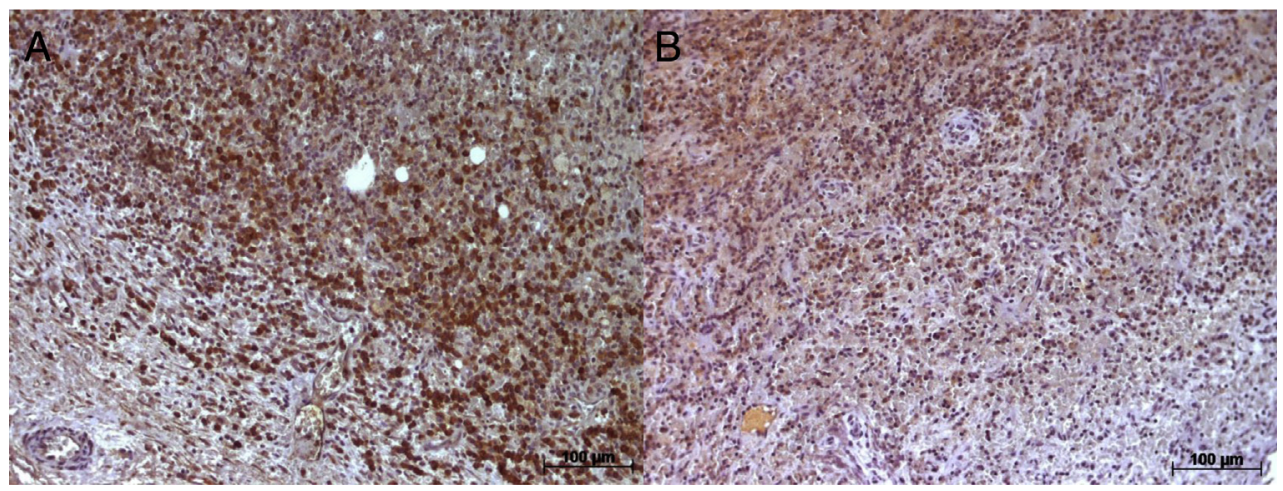

Figure 5. $(A$ and $B)$ Representative photomicrographs of immunohistochemical staining (dark brown) of positive mononuclear inflammatory cells to RANKL ( $A$ ) and OPG $(B)$ in PAP. RANKL and OPG expression in the extracellular matrix is also evidenced (original magnification, $\times 200$ ). 
RANKL

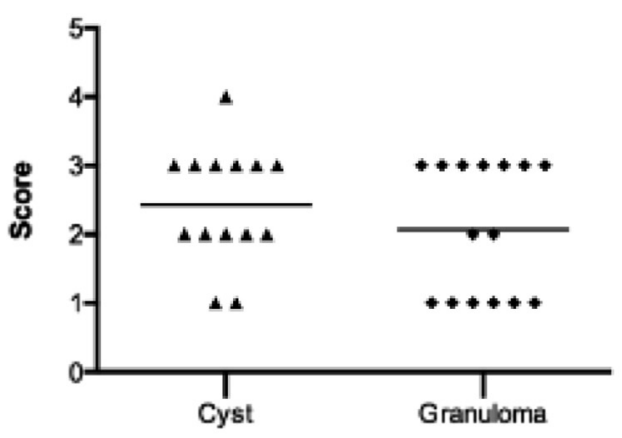

RANKL

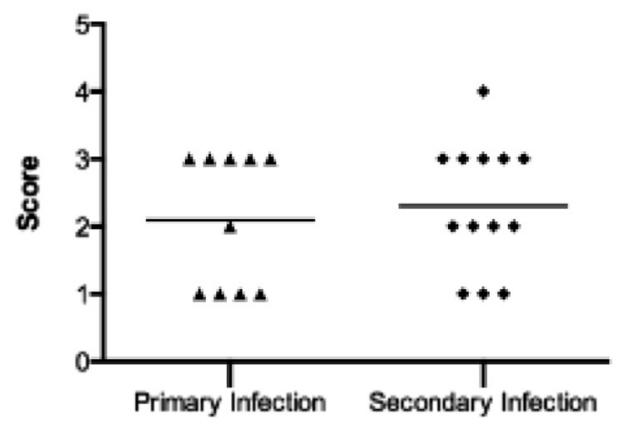

OPG

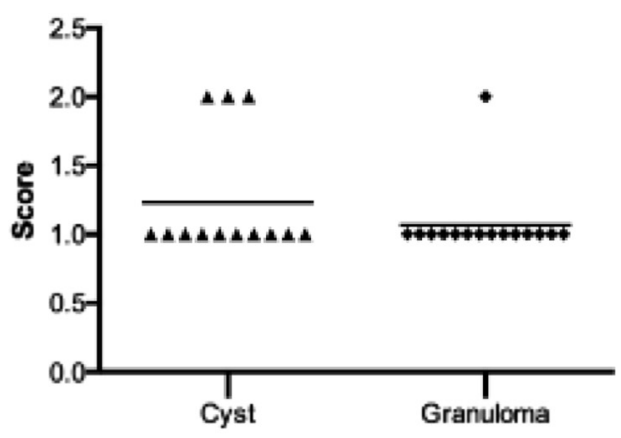

OPG

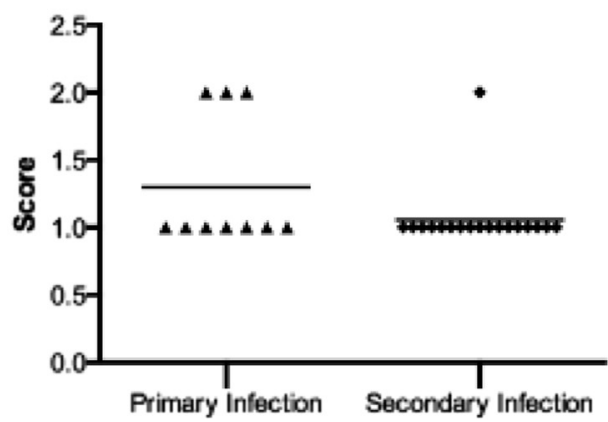

Figure 6. Immunohistochemical expression (scores) of RANKL and OPG in PAP, PPLs, cysts, and granulomas.

Inflammatory responses characterize nonspecific immediate defense mechanisms that play an important role in defense and extracellular matrix degradation through the release of metalloproteinases (3). Immune responses are specific mechanisms activated after inflammatory responses. Elevated numbers of T cells are seen in the inflammatory infiltrate of PLs (7). Intense inflammatory infiltrates are found in the presence of regulatory T lymphocytes $\left(\mathrm{FoxP}^{+}\right)$, which may indicate that they affect immunity control and prevent exacerbated inflammation. Regulatory lymphocytes may suppress $\mathrm{T}$ cells and thus reduce $\mathrm{CD}^{+}{ }^{+}$and $\mathrm{CD} 45 \mathrm{RO}^{+}$numbers (3). In this study, FoxP3 expression was higher in PAP compared with the PPL samples, which indicates that there are more Treg cells in persistent PLs than in primary lesions. Interestingly, in addition to the significant decrease in macrophages in the PAP, the present study shows a trend of reduction of all lymphocyte $T$ subtypes in persistent lesions, which may reflect a more suppressive environment in this pathology. Treg cells in PLs may lead to the inhibition of T-cell proliferation $(16,23)$ and possibly suppress Th1 response (16). Th1 response may be responsible for the induction of bone destruction and PL lesion progression because it may activate osteoclasts by RANKL (24). However, Th1 may also have inhibitory effects on bone resorption (25). Campos et al (20) observed that FoxP3 acts as a master switch governing the development and function of $\mathrm{T}$ regulatory cells, whose functions include the inhibition of immune responses and temper inflammation. Those authors found that differential methylation patterns of FoxP3 in PLs may be crucial in determining their suppressive activity and may be involved in the development of PLs.

The higher number of Treg cells in PAP found in our study may suggest the following scenarios:

1. Osteoclastic activity mediated by RANKL is decreased in PAP samples probably because of Th1 suppression.
2. Osteoclastic activity mediated by RANKL is augmented in PAP also because of Th1 suppression.

This study found a higher mRNA level of RANKL in PAP samples, with a significant imbalance between RANKL and OPG gene expressions. In contrast, no differences in the immunohistochemical expression of RANKL were found between PAP and PPL.

The expression of RANKL and OPG mRNAs is higher in human periapical granulomas than in healthy periapical tissues (26), which confirms their active role in PL progression and expansion. In periapical granulomas, the patterns of RANKL and OPG expression are heterogeneous, ranging from samples that have RANKL/OPG ratios similar to those found in sites with minimal or absent bone resorption to samples that have RANKL/OPG expression patterns that are comparable with those found in sites of active bone resorption. Clinical examinations do not determine whether periapical samples are stable or active lesions. Therefore, our study compared the profiles of RANKL/OPG expression in PLs with the expression profiles found in sites where bone resorption activity is already established, such as the compression and tension sides of teeth submitted to orthodontic forces and sites of chronic gingivitis and periodontitis (26).

Because FoxP3 expression is associated with the suppression of Th1 and Th1 response is associated with the induction of bone destruction in PLs through the RANKL, how can we explain the higher mRNA levels of RANKL found in PAP? Treg cells have a role in limiting tissue damage because of microorganism action (27), and RANKL may be stimulated by bacterial lipoteichoic acid (LTA) and bacterial lipopolysaccharide $(27,28)$. Persisting PLs after failed root canal treatment mostly have single species of gram-positive organisms (29). The isolates most commonly recovered are Enterococcus faecalis. The overall success rate of re-treatment was $74 \%$. LTA plays a significant role not only in the pathogenesis of gram-positive bacteria but also in the 
immune response of hosts (30). E. faecalis LTA has an inflammatory potential similar to that of LTAs from other gram-positive bacteria. This LTA activated macrophages that led to the induction of major inflammatory mediators such as tumor necrosis factor- $\alpha$ and nitric oxide (31). In addition, E. faecalis LTA affects the expression of RANKL and OPG in bone resorption in cases of refractory AP (27).

Conversely, Th1 may be associated with inhibitory effects on bone resorption in PAP. In this scenario, Th1 suppression may be associated with an increased expression of RANKL in PAP. RANKL and OPG are the key regulators of bone resorption in AP $(27,32)$, and RANKL may be induced by interleukin-17 in these lesions (33).

A previous study found a higher ratio of $B$ and $T$ cells ( $T$ lymphocytes $\left[\mathrm{CD}^{+}\right], \mathrm{B}\left(\mathrm{CD} 20^{+}\right)$, Thelper $\left(\mathrm{CD}^{+}\right)$, and T cytotoxic $\left.\left[\mathrm{CD}^{+}\right]\right)$in PLs of endodontically treated teeth than in those of untreated teeth (34). The proportion of T cells in the 2 experimental groups was not significantly different, which was also the case in our study. In line with our results, Rodini and Lara (7) found similar results for CD68 expression in cysts and granulomas. Those authors found no differences in CD68 between cysts and granulomas and suggested that CD68 expression might be associated with macrophages in regions of bacterial entrance. In fact, they claimed that macrophage infiltration in those PL lesions was a nonspecific inflammatory reaction associated with the pathogenesis of PL.

The mechanism underlying the persistence of some PLs is an important issue in endodontics. This study used an immunohistochemical technique to detect inflammatory cell antigens (anti-CD3, anti-CD8, anti-CD45R0, anti-FoxP3, and anti-CD68) and osteoclastogenesis markers (RANKL and OPG) and to analyze the biological characteristics of PAP and PPL. This immunohistochemical technique is an important method that may be used in several diagnostic and research conditions. Although its relevance in the search of RANKL and OPG has already been demonstrated $(3,32)$, formaldehyde fixation is critical for some epitopes, which may lead to loss of immunoreactivity depending on the resistance of the target epitope to autolytic changes (35).

This study used PL samples that persisted for more than 6 months after root canal retreatment without clinical symptoms or periapical radiolucency (PAP) and PL samples of untreated teeth (PPL). The clinical reality of periapical pathology in humans should be analyzed, and the clinical reports included in this study provided a good clinical reference for the diagnosis of PAP, which may be used to support the indication of surgical treatments as well as to accurately describe the characteristics of microscopic findings and provide precise information about cellular mechanisms.

In summary, FoxP3 expression in persistent $\mathrm{AP}$ was greater than in PPLs. There were no significant differences in the number of cells stained for CD3, CD8, and CD45R0 markers between PAP and PPL, and CD68 expression in PAP was significantly lower, which suggested a reduction of the number of macrophages. There were no differences in the immunohistochemical expression of inflammatory cell markers between periapical cysts and granulomas. RANKL and OPG immunohistochemical expressions were not significantly different between PAP and PPL or between cysts and granulomas. However, mRNA levels of RANKL and OPG genes were higher in PAP specimens, with a greater expression of RANKL than of OPG.

These finding indicate that PAP are biologically active lesions that demonstrate potential of bone resorption and are characterized by an immune-inflammatory cell profile that suggests a suppressive and regulatory environment favorable to more chronic clinical behavior.

\section{Acknowledgments}

This study was supported in part by grants from the National Council for Scientific and Technological Development
(CNPq grants \#474642/2009-7 and \#306394/2011-1 to Carlos Estrela).

The authors deny any conflicts of interest related to this study.

\section{References}

1. Nair PNR. Biology and pathology of apical periodontitis. In: Estrela C, ed. Endodontic Science, 2nd ed. São Paulo-SP, Brasil: Artes Médicas; 2009:285-347.

2. Abbas AK, Lichtman AHH, Pilai S. Cellular and Molecular Immunology, 8th ed. Philadelphia, PA: Elsevier-Saunders; 2015.

3. Bruno KF, Silva JA, Silva TA, et al. Characterization of inflammatory cell infiltrate in human dental pulpitis. Int Endod J 2010;43:1013-21.

4. Stashenko P, Teles R, D'Souza R. Periapical inflammatory responses and their modulation. Crit Rev Oral Biol Med 1998;9:498-521.

5. Al Shwaimi E, Purcell P, Kawai T, et al. Regulatory T cells in mouse periapical lesions. J Endod 2009;35:1229-33.

6. Taşman F, Er N, Ataç A, et al. CD45/isotypes expression in the immune cells of human periapical lesions. J Endod 2000;26:166-8.

7. Rodini C0, Lara VS. Study of the expression of CD68+ macrophages and CD8+ T cells in human granulomas and periapical cysts. Oral Surg Oral Med Oral Pathol Oral Radiol Endod 2001:92:221-7.

8. Liapatas S, Nakou M, Rontogianni D. Inflammatory infiltrate of chronic periradicular lesions: an immunohistochemical study. Int Endod J 2003;36:464-71.

9. Silva TA, Garlet GP, Lara VS, et al. Differential expression of chemokines and chemokine receptors in inflammatory periapical diseases. Oral Microbiol Immunol 2005; 20:310-6.

10. Matsuo T, Ebisu S, Shimabukuro Y, et al. Quantitative analysis of immunocompetent cells in human periapical lesions: correlations with clinical findings of the involved teeth. J Endod 1992;18:497-500.

11. Peixoto RF, Pereira JS, Nonaka CF, et al. Immunohistochemical analysis of FoxP3+ cells in periapical granulomas and radicular cysts. Arch Oral Biol 2012;57:1159-64.

12. Kawashima N, Suzuki N, Yang G, et al. Kinetics of RANKL, RANK and OPG expressions in experimentally induced rat periapical lesions. Oral Surg Oral Med Oral Pathol Oral Radiol Endod 2007;103:707-11.

13. Boyle WJ, Simonet WS, Lacey DL. Osteoclast differentiation and activation. Nature 2003; $423: 337-42$.

14. Silva TA, Garlet GP, Fukada SY, et al. Chemokines in oral inflammatory diseases: apical periodontitis and periodontal disease. J Dent Res 2007;86:306-19.

15. Maruyama K, Takada Y, Ray N, et al. Receptor activator of NF-kappa B ligand and osteoprotegerin regulate proinflammatory cytokine production in mice. J Immunol 2006;177:3799-805.

16. Fukada SY, Silva TA, Garlet GP, et al. Factors involved in the T helper type 1 and type 2 cell commitment and osteoclast regulation in inflammatory apical diseases. Oral Microbiol Immunol 2009;24:25-31.

17. Sato K, Suematsu A, Okamoto K, et al. Th17 functions as an osteoclastogenic helper T cell subset that links T cell activation and bone destruction. J Exp Med 2006;203: 2673-82.

18. Zhang X, Peng B. Immunolocalization of receptor activator of NF kappa B ligand in rat periapical lesions. J Endod 2005;31:574-7.

19. Fontenot JD, Rudensky AY. A well adapted regulatory contrivance: regulatory T cell development and the forkhead family transcription factor Foxp3. Nat Immunol 2005;6:331-7.

20. Campos K, Franscisconi CF, Okehie V, et al. FOXP3 DNA methylation levels as a potential biomarker in the development of periapical lesions. J Endod 2015;41:212-8.

21. Gori S, Sidoni A, Colozza M, et al. EGFR, pMAPK, pAkt and PTEN status by immunohistochemistry: correlation with clinical outcome in HER2-positive metastatic breast cancer patients treated with trastuzumab. Ann Oncol 2009:20:648-54.

22. Hiraishi Y, Wada T, Nakatani K, et al. Immunohistochemical expression of EGFR and p-EGFR in oral squamous cell carcinomas. Pathol Oncol Res 2006;12:87-91.

23. Andrade AL, Nonaka CF, Gordon-Nunez MA, et al. Immunoexpression of interleukin 17, transforming growth factor beta1, and forkhead box P3 in periapical granulomas, radicular cysts, and residual radicular cysts. J Endod 2013;39:990-4.

24. Brito IC, Teles FR, Teles RP, et al. T-lymphocyte and cytokine expression in human inflammatory periapical lesions. J Endod 2012;38:481-5.

25. Alayan J, Ivanovski S, Farah CS. Alveolar bone loss in T helper 1/T helper 2 cytokinedeficient mice. J Periodont Res 2007;42:97-103.

26. Menezes R, Garlet TP, Letra A, et al. Differential patterns of receptor activator of nuclear factor kappa B ligand/osteoprotegerin expression in human periapical granulomas: possible association with progressive or stable nature of lesions. J Endod 2008:34:932-8.

27. Chen J, Du J, Wang F, et al. Effects of Enterococcus faecalis lipoteichoic acid on receptor activator of nuclear factor- $\kappa \mathrm{B}$ ligand and osteoprotegerin expression in periodontal ligament fibroblasts. Int Endod J 2014;47:163-72. 


\section{Basic Research-Biology}

28. Wada T, Nakashima T, Oliveira-dos-Santos AJ, et al. The molecular scaffold Gab2 is a crucial component of RANK signaling and osteoclastogenesis. Nat Med 2005;11:394-9.

29. Sundqvist G, Figdor D, Persson S, Sjögren U. Microbiologic analysis of teeth with failed endodontic treatment and the outcome of conservative re-treatment. Oral Surg Oral Med Oral Pathol Oral Radiol Endod 1998;85:86-93.

30. Ginsburg I. Role of lipoteichoic acid in infection and inflammation. Lancet Infect Dis 2002;2:171-9.

31. Baik JE, Ryu YH, Han JY, et al. Lipoteichoic acid partially contributes to the inflammatory responses to Enterococcus faecalis. J Endod 2008;34:975-82.
32. Boyce BF, Xing L. Functions of RANKL/RANK/OPG in bone modeling and remodeling. Arch Biochem Biophys 2008;473:139-46.

33. Marçal JR, Samuel R0, Fernandes D, et al. T-helper cell type 17/regulatory T-cell immunoregulatory balance in human radicular cysts and periapical granulomas. J Endod 2010;36:995-9.

34. Alavi AM, Gulabivala K, Speight PM. Quantitative analysis of lymphocytes and their subsets in periapical lesions. Int Endod J 1998;31:233-41.

35. Matos LL, Trufelli DC, Matos MGL, Pinhal MAS. Immunohistochemistry as an important tool in biomarkers detection and clinical practice. Biomark Insights 2010;5:9-20. 